\title{
MicroRNAs, innate immunity and ventricular rupture in human myocardial infarction
}

\author{
Nina Zidar ${ }^{\mathrm{a}, *}$, Emanuela Boštjančič ${ }^{\mathrm{b}}$, Damjan Glavač ${ }^{\mathrm{b}}$ and Dušan Štajer ${ }^{\mathrm{c}}$ \\ anstitute of Pathology, Faculty of Medicine, University of Ljubljana, Ljubljana, Slovenia \\ ${ }^{\mathrm{b}}$ Department of Molecular Genetics, Institute of Pathology, Faculty of Medicine, University of Ljubljana, \\ Ljubljana, Slovenia \\ ${ }^{\mathrm{c}}$ Centre for Intensive Internal Medicine, University Medical Centre, Ljubljana, Slovenia
}

\begin{abstract}
MicroRNAs are non-coding RNAs, functionioning as post-transcriptional regulators of gene expression. Some microRNAs have been demonstrated to play a role in regulation of innate immunity. After myocardial infarction (MI), innate immunity is activated leading to an acute inflammatory reaction. There is evidence that an intense inflammatory reaction might contribute to the development of ventricular rupture (VR) after MI.

Using real-time PCR, we analysed the expression of $m i R-146 a$, miR-150, and miR-155 in autopsy samples of infarcted heart tissue from 50 patients with MI (23 with VR and 27 without VR).

An altered expression of all three microRNAs was found in MI compared to the normal hearts. Comparing MI patients with VR and those without VR, we found $m i R-146 a$ up-regulation, and $m i R-150$ and $m i R-155$ down-regulation in patients with VR.

In conclusion, our study demonstrated an altered expression of $m i R-146 a, m i R-150$, and $m i R-155$ in MI compared to the normal hearts. These microRNAs are involved in regulation of the innate immunity. Differential expression of these microRNAs in MI patients with VR in comparison to those without VR provides further evidence that innate immunity resulting in an intense inflammatory reaction plays an important role in the pathogenesis of VR after MI in humans.
\end{abstract}

Keywords: Myocardial infarction, ventricular rupture, pathogenesis, inflammation, innate immunity, microRNA

\section{Introduction}

Experimental studies have shown that rupture of the ventricular wall (ventricular rupture, VR) in a mouse model of myocardial infarction (MI) occurs 2-6 days after $\mathrm{MI}$ and that it is associated with the size of MI and the extent of the regional and systemic inflammatory response [1,2]. Similar findings have been observed in our clinicopathologic study of VR in human MI [3]. We analysed the density of interstitial neutrophil infiltration in autopsy samples of infarcted heart tissue from 110 patients (50 patients with VR, and 60 patients without VR). We found a significantly more intensive interstitial neutrophil infiltration of the infarcted myocardium in patients with VR compared to the patients without VR,

*Corresponding author: Nina Zidar, Institute of Pathology, Faculty of Medicine, University of Ljubljana, Korytkova 2, 1000 Ljubljana, Slovenia. Tel.: +386 1543 7149; Fax: +386 1543 7101; E-mail: nina.zidar@mf.uni-lj.si. who died 2-7 after the onset of MI. In patients with VR, arterial hypertension was significantly more frequent than in patients without VR. These findings suggest that inflammatory response mediated by neutrophils might play an important role in the pathogenesis of the VR also in humans, particularly in those with arterial hypertension.

Neutrophils accumulate in the infarcted myocardium as a part of the innate immune response, which has been recently shown to be modulated by microRNAs. MicroRNAs are short ( 22 nucleotides long), single-stranded and non-coding RNAs that function as post-transcriptional regulators of gene expression [4, 5]. Since being defined, it has been shown that they are involved in the regulation of a wide variety of cellular processes, including apoptosis, differentiation, and proliferation. As a consequence, they contribute to various physiological and pathological conditions, including immune response [6] and cardiovascular diseases [7]. Some microRNAs, e.g. $m i R-146 a, m i R-150$, and $m i R-155$, have been described to play a role in the 
regulation of the innate immune response [8,9]. To deepen our understanding of the postulated role of the innate immune response in the pathogenesis of VR after MI, we analysed the expression of these microRNAs in the infarcted heart tissue samples from patients with MI with VR in comparison to those without VR.

\section{Materials and methods}

\subsection{Patients and tissue sections}

We examined the expression profile of miR-146a, $m i R-150$ and $m i R-155$ in 50 patients (27 males and 23 females, aged 56-94 years, mean age $72.6 \pm 9.5$ ) with MI, who died of VR or other complications. MI was diagnosed clinically by symptoms and/or electrocardiographic changes, and confirmed by elevated plasma levels of markers of cardiac necrosis. The heart tissue samples were obtained at autopsy within 24 hours after death, and were taken from the infarcted region and border zone. Tissue samples were fixed in $10 \%$ buffered formalin and embedded in paraffin. The duration of MI at the time of death was estimated on the basis of pathohistological changes and clinical data [3], and all cases of MI were divided into two groups: up to 24 hours after onset of clinical symptoms (MI1) and 2-7 days after onset of clinical symptoms (MI2). There were 25 patients with MI1 (15 without VR and 10 with VR), and 25 patients with MI2 (12 without VR and 13 with VR). Diabetes and arterial hypertension was recorded in 35 and 37 patients, respectively. Twentyfour patients had received reperfusion treatment (11 with MI1 and 14 with MI2).

The control group consisted of autopsy heart tissue from 10 healthy adults (6 males and 4 females, aged $18-35$, mean age $27.3 \pm 5.91$ ) who died in accidents. Tissue samples were fixed in $10 \%$ buffered formalin and embedded in paraffin. Post mortem delay did not exceed 24 hours, and there was no macroscopical or microscopical evidence of disease at autopsy.

The study conforms with the Code of Ethics of the World Medical Association (Helsinki Declaration), printed in the British Medical Journal (18 July 1964). It was approved by the National Medical Ethics Committee of Slovenia (ref. No 39/01/08).

\subsection{RNA isolation}

Tissue samples were cut at $10 \mu \mathrm{m}$ from formalinfixed paraffin-embedded (FFPE) tissue blocks using a microtome. Six to eight $10-\mu \mathrm{m}$ sections were used for the isolation procedure. Total RNA isolation was performed using a miRNeasy FFPE kit (Qiagen) according to the manufacturer's protocol. All the reagents were from Qiagen, except where otherwise indicated. Briefly, $1 \mathrm{ml}$ of Xylene (Merck) was added for deparaffinisation, followed by brief vortexing and centrifugation. After the ethanol-washing step, pellets were air-dried and digestion with Proteinase $\mathrm{K}$ was performed at $55^{\circ} \mathrm{C}$ for $15 \mathrm{~min}$, followed by $15 \mathrm{~min}$ incubation at $80^{\circ} \mathrm{C}$ in order to partially reverse formaldehyde modification of nucleic acid. After the gDNA elimination step, $100 \%$ ethanol (Merck) was added to the samples and the mixture was transferred to an RNeasy MinElute spin column. After two washing steps, the RNA was eluted in $30 \mu$ l of nuclease-free water. The concentration of RNA extracted was measured using NanoDrop-1000 (Thermo Scientific) and tested for $\mathrm{UV} / \mathrm{vis}$ ratios. The $\mathrm{A}_{260} / \mathrm{A}_{230} \mathrm{~nm}$ intensity ratio needs to be above 1.0 and $\mathrm{A}_{260} / \mathrm{A}_{280}$ ratio needs to be above 1.8 . The integrity and presence of small RNAs ( $<200$ nucleotides) was analyzed on a Bioanalyzer 2100 (Agilent, USA). Using the Small RNA Assay and Small RNA Ladder as reference we obtained the detailed view of the RNA of 6-150 nt in range and look for the presence of $\sim 22 \mathrm{nt}$ long RNA molecules.

\subsection{DNA digestion step and no-reverse transcriptase control}

RNA obtained from FFPE heart tissue samples was treated with DNAse I prior to qPCR to eliminate the possibility of unspecific detection of double-stranded DNA amplified during the qPCR reaction. Up to $1 \mu \mathrm{g}$ $(5 \mu \mathrm{l})$ of RNA contaminated with genomic DNA was used, treated with a mix containing $2 \mu \mathrm{l} 10 \mathrm{x}$ DNase buffer (RDD buffer), 10 units RNase inhibitor, $0.5 \mathrm{Ku}$ nitz units DNase I and filled with RNase-free water to obtain a $20 \mu \mathrm{l}$ reaction mixture. After $30 \mathrm{~min}$ incubation step at $37^{\circ} \mathrm{C}, 2 \mu \mathrm{l} 140 \mathrm{mM}$ EDTA (Sigma) was added to the reaction and incubated for $5 \mathrm{~min}$ at $65^{\circ} \mathrm{C}$ to inactivate DNase I. The concentration was measured using NanoDrop-1000. The step of no-reverse transcriptase control included all the qPCR reagents with the primers and the RNA treated with DNAase I, without the reverse transcription step. If the product was amplified, it indicated that there was still some genomic DNA contamination. 


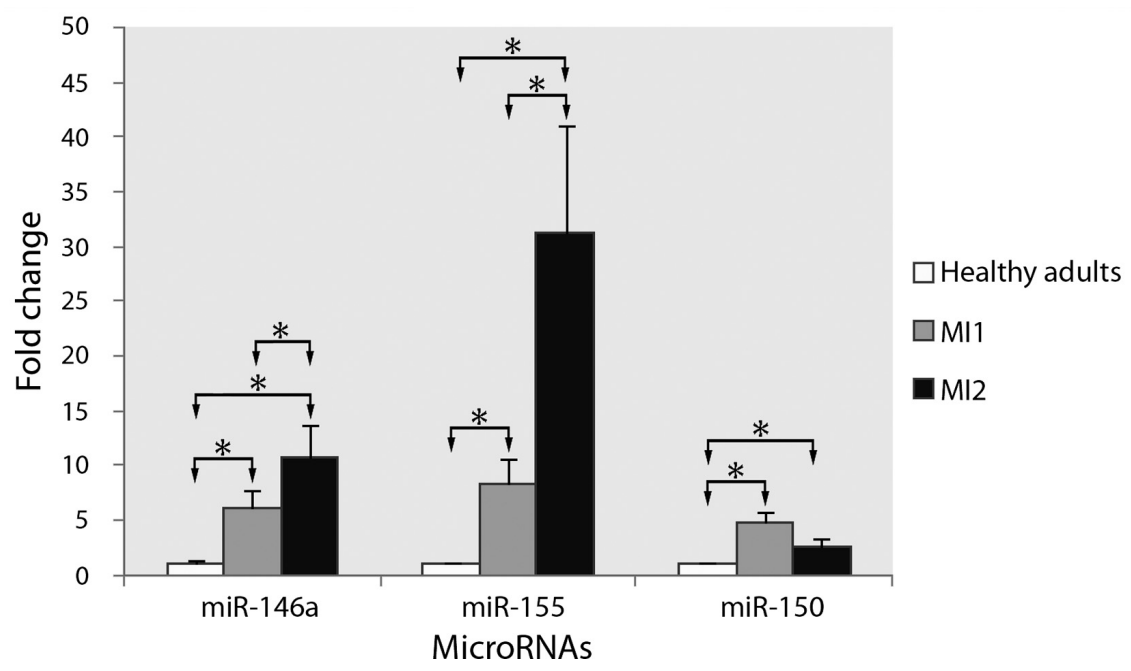

Fig. 1. Fold change differences in expression of $m i R-146 a$, $m i R-155$ and $m i R-150$ in human myocardial infarction compared to healthy adult hearts. Legend: MI1, up to 24 hours after onset of MI; MI2, 2-7 days after onset of MI; * , statistically significant fold change in microRNAs expression.

\subsection{Reverse transcription and $q P C R$ using miScript system}

A miScript reverse transcription kit was used for reverse transcription. Briefly, a 15- $\mu$ l reaction master mix was created, containing $100 \mathrm{ng}$ of total RNA, $3 \mu \mathrm{l} 5 \mathrm{x}$ miScript RT buffer, $0.75 \mu \mathrm{l}$ miScript reverse transcriptase mix and 10 units $(0.33 \mu \mathrm{l})$ RNase inhibitor. After incubation for $60 \mathrm{~min}$ at $37^{\circ} \mathrm{C}$ and $5 \mathrm{~min}$ at $95^{\circ} \mathrm{C}$, the cDNA was diluted 10-fold, and $4.5 \mu \mathrm{l}$ was used for each qPCR reaction. The $15 \mu \mathrm{l}$ PCR master mix contained $7.5 \mu 12 x$ QuantiTect SYBR Green PCR Master Mix, $1.5 \mu \mathrm{l} 10 \mathrm{x}$ miScript universal primer and $1.5 \mu \mathrm{l} 10 \mathrm{x}$ miScript Primer Assay. The reaction was performed under the following thermal cycling conditions: initial denaturation at $95^{\circ} \mathrm{C}$ for 15 minutes; cycling 40 times at $94^{\circ} \mathrm{C}$ for 15 seconds, at $55^{\circ} \mathrm{C}$ for 30 seconds and at $70^{\circ} \mathrm{C}$ for 15 seconds. The signal was collected at the end point of every cycle. All the qPCR reactions were performed in duplicate or triplicate on ABI 7900 (Applied Biosystems). Following amplification, melting curves analysis of PCR products was performed to verify specify and identity. Melting curves were acquired on the SYBR channel using a ramping rate of $1^{\circ} \mathrm{C} / 60 \mathrm{~s}$ for $60-95^{\circ} \mathrm{C}$. $R N U 6 B$ was used as the internal control gene, according to the manufacturer's protocol.

\subsection{Statistical analysis}

The $2^{-\Delta \Delta C t}$ method was used to present the relative gene expression obtained from qPCR analysis, where- by the fold changes of the tested groups were calculated relative to the calibrator group as previously described [10]. The calculated $\Delta \mathrm{Ct}$ (difference between $\mathrm{Ct}$ of miRNA of interest and $\mathrm{Ct}$ of $R N U 6 B$ ) in different groups were compared to the $\Delta \mathrm{Ct}$ of the control group (calibrator group) and tested for statistical significance using the Mann-Whitney test for independent groups of samples, with a cut off point at $p<0.05$. To calculate the differences in miRNA expression between the defined groups of patients, and correlations with clinical data, the following statistical tests were used: MannWhitney test, Kruskal-Wallis test and Sperman's Rho correlation coefficient. All the statistical tests were included in SPSS analytical software ver.17 (SPSS Inc., Chicago, Ill., USA).

\section{Results}

We found differences in expression patterns of $m i R$ $146 a$, $m i R-155$ and $m i R-150$ within the defined timewindows (up to $24 \mathrm{~h}$ and 2-7 days after MI onset) when compared to healthy adults. All tested microRNAs were significantly up-regulated in MI1 and MI2. The miR-150 showed the lowest expression in MI2, whereas miR-146a and miR-155 showed the peak expression in MI2. For miR-146a and miR-155, a significant difference between MI1 and MI2 was found. Results are summarized in Fig. 1.

We further examined if there is any correlation between the tested microRNAs and the presence of VR. 


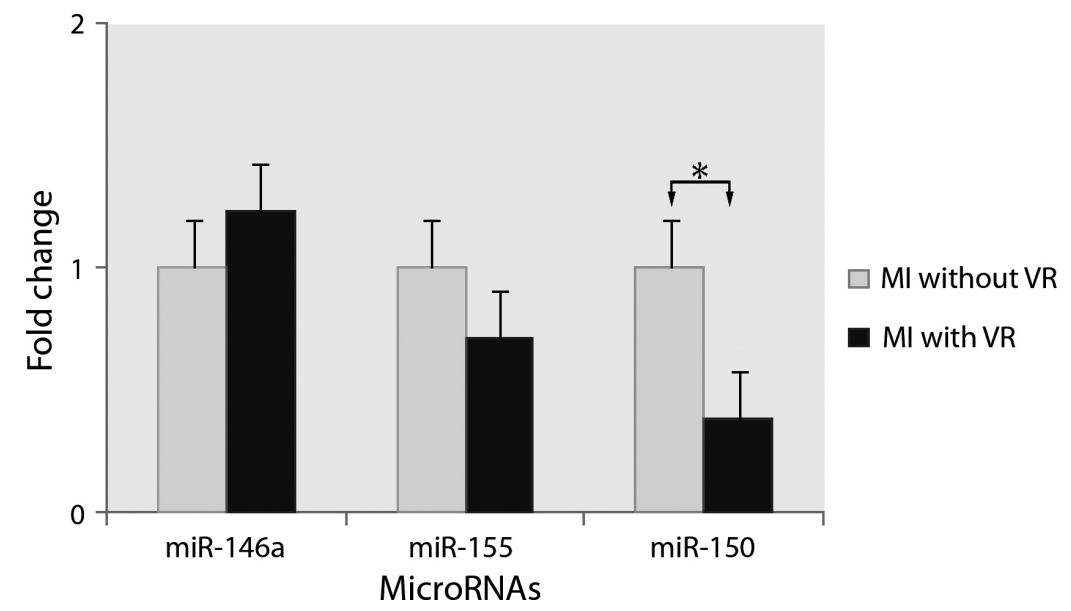

Fig. 2. Fold change differences in expression of miR-146a, miR-155 and miR-150 in myocardial infarction with and without ventricular rupture. Legend: MI, myocardial infarction; VR, ventricular rupture; *, statistically significant fold change in microRNAs expression.

We observed a significant correlation between $m i R$ $146 a, m i R-150$ and $m i R-155$ expression. There was also an inverse correlation between $m i R-150$ and the presence of VR $(p=0.022)$. In patients with VR compared to those without VR, $m i R-150$ was significantly down-regulated, whereas $m i R-155$ down-regulation and miR146a up-regulation did not reach the statistical significance. Results are summarized in Fig. 2.

Comparing the expression of $m i R-146 a, m i R-150$ and $m i R-155$ in patients with VR and those without VR in both MI groups, we found miR-146a up-regulation in patients with VR only in MI2. No difference in $m i R$ $146 a$ expression was observed in MI1 between patients with and those without VR. In contrast, we found $m i R$ 155 and $m i R-150$ down-regulation in patients with VR compared to patients without VR in both MI1 and MI2. The strongest correlation was observed for $m i R-150$. The results are summarized in Fig. 3. We also observed statistically significant differences in $m i R-146 a$ and $m i R-155$ expression in patients with VR in MI1 compared to those with VR in MI2. Similar changes were observed for $m i R-155$ expression in patients without VR in MI1 compared to those without VR in MI2. Results are summarized in Fig. 3 and Table 1.

When we analyzed the expression of microRNAs in relation to the reperfusion treatment, we found that only $m i R-155$ is differentially expressed if we compare MI1 and MI2 patients $(p=0.015)$. MicroRNA miR155 was up-regulated in patients with MI2 that received reperfusion treatment when compared to patients with MI2 that did not receive reperfusion treatment. The results are summarized in Table 2.
Table 1

Expression of microRNAs $m i R-146 a, m i R-155$ and $m i R-150$ in patients with myocardial infarction, in relation to ventricular rupture

\begin{tabular}{clccc}
\hline deltaCt & & $m i R-146 a$ & $m i R-155$ & $m i R-150$ \\
\hline \multirow{2}{*}{ MI1 } & without VR & $6.16 \pm 1.61$ & $6.31 \pm 1.60$ & $1.91 \pm 2.17$ \\
& with VR & $6.19 \pm 1.13$ & $7.19 \pm 1.07$ & $3.26 \pm 1.50$ \\
MI2 & without VR & $5.72 \pm 1.19$ & $4.56 \pm 2.45$ & $2.82 \pm 1.39$ \\
& with VR & $5.08 \pm 1.67$ & $4.99 \pm 2.15$ & $3.82 \pm 2.56$ \\
\hline
\end{tabular}

Legend: MI1, up to 24 hours after onset of M I; MI2, 2-7 days after onset of MI; VR, ventricular rupture.

\section{Discussion}

In this study, we found an altered expression of microRNAs $m i R-146 a$, $m i R-150$ and $m i R-155$ in the infarcted heart tissue samples from patients with MI in comparison to the samples of the normal human hearts. The expression of $m i R-146 a$ and $m i R-155$ was highest in patients 2 to 7 days after the onset of MI, whereas the expression of $m i R-150$ showed the lowest expression in patients 2 to 7 days after the onset MI. These findings are consistent with the postulated role of these microRNAs in the innate immunity [11], as well as with the dynamics of the acute inflammatory response in MI, which is believed to reach a peak during the first week after MI [3].

In MI, innate immune mechanisms are activated by necrotic cardiomyocytes, which release their intracellular contents, resulting in an intense inflammatory reaction [12]. The innate immune mechanisms comprise the Toll-like receptor-mediated pathways, the complement cascade and reactive oxygen generation, induction of nuclear factor (NF)- $\kappa \mathrm{B}$ activation and upregulation of chemokine and cytokine synthesis, leading to the accumulation of neutrophils and other in- 
Table 2

Expression of microRNAs miR-146a, miR-155 and miR-150 in patients with myocardial infarction, in relation to ventricular rupture and reperfusion treatment

\begin{tabular}{llclc}
\hline delta Ct & & $m i R-146 a$ & $m i R-155$ & $m i R-150$ \\
\hline MI1 & reperfusion & $6.30 \pm 1.25$ & $6.88 \pm 1.20^{*, a}$ & $2.39 \pm 1.72$ \\
& no reperfusion & $6.09 \pm 1.51$ & $6.54 \pm 1.62^{*}$ & $2.63 \pm 2.09$ \\
MI2 & reperfusion & $5.50 \pm 1.37$ & $4.65 \pm 2.53^{*, a}$ & $3.03 \pm 2.59$ \\
& no reperfusion & $5.30 \pm 1.69$ & $5.18 \pm 1.96^{*}$ & $3.16 \pm 1.83$ \\
\multirow{2}{*}{ with VR } & reperfusion & $5.60 \pm 1.21$ & $6.50 \pm 1.84$ & $3.44 \pm 2.10$ \\
& no reperfusion & $5.80 \pm 1.67$ & $5.80 \pm 2.16$ & $3.22 \pm 1.81$ \\
\multirow{2}{*}{ ithout VR } & reperfusion & $6.08 \pm 1.47$ & $5.50 \pm 2.69$ & $2.12 \pm 1.95$ \\
& no reperfusion & $5.76 \pm 1.59$ & $5.62 \pm 1.49$ & $2.09 \pm 1.95$ \\
\hline
\end{tabular}

Legend: MI1, up to 24 hours after onset of MI; MI2, 2-7 days after onset of MI; VR, ventricular rupture; reperfusion, receiving reperfusion treatment.

*,Statistical significance in expression of miR-155 between the groups $(p=0.014$ and $p=0.015$, Kruskal-Wallis test and ANOVA, respectively; $\rho_{\mathrm{s}}=0.431$, $p=0.002$, Spearman's Rho); ${ }^{\text {a }}$,Statistical significance in expression of miR-155 between MI1 and MI2 that received reperfusion treatment ( $p=0.013)$, Student's t-test.

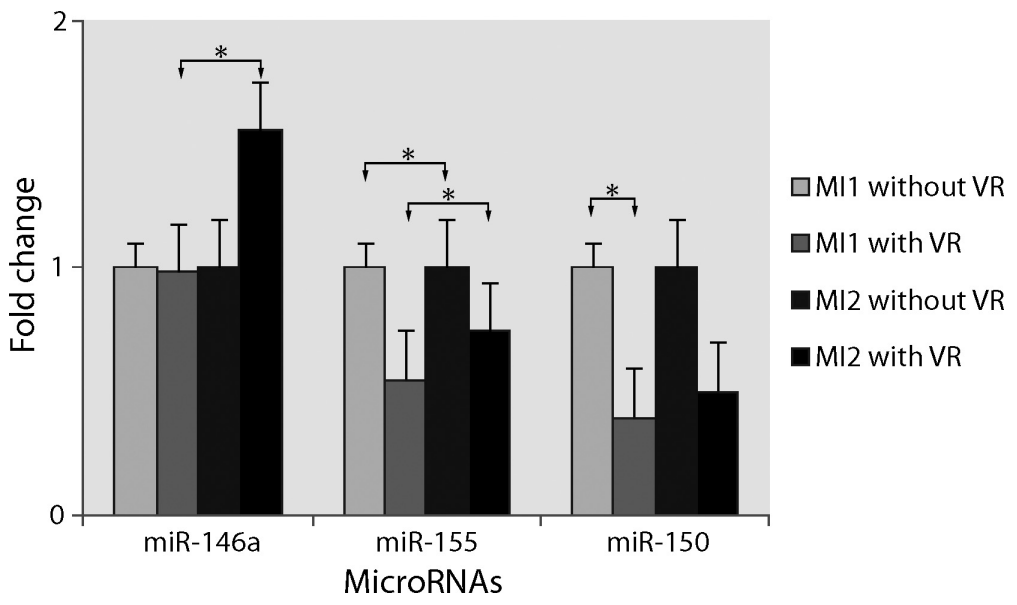

Fig. 3. Fold change differences in expression of $m i R-146 a$, $m i R-155$ and $m i R-150$ in myocardial infarction with and without ventricular rupture. Statistical analysis of miRNA expression was performed based on $\Delta \mathrm{Ct}$, which was calculated as the difference between Ct of miRNA of interest and $\mathrm{Ct}$ of RNU6B. Results of microRNAs expression are showed separately for MI1 and MI2, where the relative microRNAs expression is calibrated as follows: miRNA expression in MI1 patients with VR relatively to MI1 patients without VR and miRNA expression in MI2 patients with VR relatively to MI2 patients without VR. Legend: MI1, up to 24 hours after onset of MI; MI2, 2-7 days after onset of MI; VR, ventricular rupture; *, statistically significant fold change in microRNAs expression.

flammatory cells. Many of these processes have been demonstrated to be regulated by microRNAs, including $m i R-146 a$, $m i R-150$, and $m i R-155$ [8,13-16], and only a few targets have been directly identified for these microRNAs [17]. $m i R-146 a$ and $m i R-155$, for example, are induced by toll-like receptors (TLR2, 3, 4 and 9), by interleukin-1 (IL-1) and tumor necrosis factor $\alpha$ (TNF- $\alpha$ ), and upon activation of NF$\kappa \mathrm{B}$ [18]. Their known targets include interleukin8 (IL-8), RANTES (CCL5), interleukin-1 receptorassociated kinase 2 (IRAK2) and tumor necrosis factor receptor-associated factor 6 (TRAF6) [8,14,19], c/ebp Beta and granulocyte colony-stimulating factor (G-CSF) [20], metalloproteinases [8], and many oth- ers [17]. MicroRNA miR-150 was also described to be down-regulated in the innate immune response (sepsis), but its targets in this condition are not known yet [21].

Innate immunity probably plays a key role in the early stages of repair after MI, but, if too intensive, might also enhance the ishemic/reperfusion injury. The regulation of the innate immunity might therefore be crucial in determining the intensity of the inflammatory reaction and, speculatively, contribute to the development of VR after MI [1-3]. When we compared patients with MI with VR and those without VR, we found down-regulation of $m i R-150$ and $m i R-155$, and up-regulation of $m i R-146 a$ in patients with VR. Downregulation of $m i R-150$ and $m i R-155$ was the strongest 
in patients with VR who died within 24 hours after the onset of MI, whereas up-regulation of $m i R-146 a$ was the strongest in patients with VR who died 2 to 7 days after the onset of MI.

These findings are in accordance with previous studies which have shown that up-regulation of miR-146a is associated with proinflammatory cytokines and the $\mathrm{NF}-\kappa \mathrm{B}$ signalling $[13,22]$.

Similarly, down-regulation of $m i R-150$ and $m i R-155$ has been demonstrated to be associated with the induction of inflammation [23,24]. Down-regulation of $m i R-155$ was among other effects associated with the elevated expression of matrix metalloproteinases [23, 24]. Intensive inflammation [2,3] and elevated metalloproteinases with excessive degradation of the extracellular matrix [25-27] have been implicated in the development of VR after MI in experimental models and human MI. Our finding of down-regulation of $m i R-150$ and $m i R-155$ is thus consistent with the hypothesis that intensive inflammatory reaction, particularly in patients who received reperfusion treatment, plays an important role in the pathogenesis of VR after MI.

\section{Conclusion}

Our study demonstrated an altered expression of $m i R-146 a, m i R-150$, and $m i R-155$ in patients with MI in comparison to the normal hearts. These microRNAs have been postulated to be involved in the regulation of the innate immune response. Differential expression of these microRNAs in MI patients with VR in comparison to those without VR provides further evidence that innate immunity resulting in an intense inflammatory reaction plays an important role in the pathogenesis of the VR after MI in humans.

\section{References}

[1] X.M. Gao, Q. Xu, H. Kiriazis, A.M. Dart and X.J. Du, Mouse model of post-infarct ventricular rupture: time course, strainand gender-dependency, tensile strength, and histopathology, Cardiovasc Res 65 (2005), 469-477.

[2] X.M. Gao, Z. Ming, Y. Su, L. Fang, H. Kiriazis, Q. Xu, A.M. Dart and X.J. Du, Infarct size and post-infarct inflammation determine the risk of cardiac rupture in mice, Int $J$ Cardiol 143 (2010), 20-28.

[3] N. Zidar, J. Jeruc, J. Balažic and D. Štajer, Neutrophils in human myocardial infarction with rupture of the free wall, Cardiovascular Pathol 14 (2005), 247-250.

[4] R.S. Pillai, MicroRNA function: multiple mechanisms for a tiny RNA? RNA 11 (2005), 1753-1761.
[5] R.S. Pillai, S.N. Bhattacharyya and W. Filipowicz, Repression of protein synthesis by miRNAs: how many mechanisms? Trends Cell Biol 17 (2007), 118-126.

[6] E. Tsitsiou and M.A. Lindsay, MicroRNAs and the immune response, Curr Opin Pharmacol 9 (2009), 514-520.

[7] E. Scalbert and A. Bril, Implication of microRNAs in the cardiovascular system, Curr Opin Pharmacol 8 (2008), 181188.

[8] F.J. Sheedy and L.A. O'Neill, Adding fuel to fire: microRNAs as a new class of mediators of inflammation, Ann Rheum Dis 67(Suppl 3) (2008), 50-55.

[9] R.M. O'Connell, A.A. Chaudhuri, D.S. and D. Baltimore, Physiological and pathological roles for microRNAs in the immune system, Nat Rev Immunol 10 (2010), 111-122.

[10] E. Boštjančič, N. Zidar and D. Glavač, MicroRNA microarray expression profiling in human myocardial infarction, Dis Markers 27 (2009), 255-268.

[11] J.E. Dahlberg and E. Lund, Micromanagement during the innate immune response, Sci STKE 387 (2007), pe25.

[12] N.G. Frangogiannis, The immune system and cardiac repair, Pharmacol Res 58 (2008), 88-111.

[13] K.D. Taganov, M.P. Boldin, K.J. Chang and D. Baltimore, NF-kappaB-dependent induction of microRNA miR-146, an inhibitor targeted to signaling proteins of innate immune responses, Proc Natl Acad Sci USA 103 (2006), 12481-12486.

[14] Y. Takahashi, M. Satoh, Y. Minami, T. Tabuchi, T. Itoh and M. Nakamura, Expression of miR-146a/b is associated with the Toll-like receptor 4 signal in coronary artery disease: effect of renin-angiotensin system blockade and statins on miRNA146a/b and Toll-like receptor 4 levels, Clin Sci (London) 119 (2010), 395-405.

[15] C.E. McCoy, F.J. Sheedy, J.E. Qualls, S.L. Doyle, S.R. Quinn, P.J. Murray and L.A. O'Neill, IL-10 inhibits miR-155 induction by toll-like receptors, J Biol Chem 285 (2010), 2049220498.

[16] G. Teng and F.N. Papavasiliou, Shhh! Silencing by microRNA-155, Philos Trans $R$ Soc Lond B Biol Sci 364 (2009), 631-637.

[17] M.P. Gantier, New perspectives in microRNA regulation of innate immunity, J Interferon Cytokine Res 30 (2010), 283289.

[18] M. van de Vrie, S. Heymans and B. Schroen, MicroRNA involvement in immune activation during heart failure, Cardiovasc Drugs Ther 25 (2011), 161-170.

[19] W.M. Schmidt, A.O. Spiel, B. Jilma, M. Wolzt and M. Müller, In vivo profile of the human leukocyte microRNA response to endotoxemia, Biochem Biophys Res Commun 380 (2009), $437-441$.

[20] J. Worm, J. Stenvang, A. Petri, K.S. Frederiksen, S. Obad, J. Elmén, M. Hedtjärn, E.M. Straarup, J.B. Hansen, S. Kauppinen, Silencing of microRNA-155 in mice during acute inflammatory response leads to derepression of c/ebp Beta and down-regulation of G-CSF, Nucleic Acids Res 37 (2009), 5784-5792.

[21] C. Vasilescu, S. Rossi, M. Shimizu, S. Tudor, A. Veronese, M. Ferracin, M.S. Nicoloso, E. Barbarotto, M. Popa, O. Stanciulea, M.H. Fernandez, D. Tulbure, C.E. Bueso-Ramos, M. Negrini and G.A. Calin, MicroRNA fingerprints identify miR150 as a plasma prognostic marker in patients with sepsis, PLoS One 4 (2009), e7405.

[22] L. Li, X.P. Chen and Y.J. Li, MicroRNA-146a and human disease, Scand J Immunol 71 (2010), 227-231.

[23] I. Faraoni, F.R. Antonetti, J. Cardone and E. Bonmassar, miR155 gene: a typical multifunctional microRNA, Biochim Bio- 
phys Acta 1792 (2009), 497-505.

[24] S.W. Jones, G. Watkins, N. Le Good, S. Roberts, C.L. Murphy, S.M. Brockbank, M.R. Needham, S.J. Read and P. Newham, The identification of differentially expressed microRNA in osteoarthritic tissue that modulate the production of TNF-alpha and MMP13, Osteoarthritis Cartilage 17 (2009), 464-472.

[25] S. Heymans, A. Luttun, D. Nuyens, G. Theilmeier, E. Creemers, L. Moons, G.D. Dyspersin, J.P. Cleutjens, M. Shipley, A. Angellilo, M. Levi, O. Nübe, A. Baker, E. Keshet, F. Lupu, J.M. Herbert, J.F. Smits, S.D. Shapiro, M. Baes, M. Borgers, D. Collen, M.J. Daemen and P. Carmeliet, Inhibition of plasminogen activators or matrix metalloproteinases pre- vents cardiac rupture but impairs therapeutic angiogenesis and causes cardiac failure, Nat Med 5 (1999), 1135-1142.

[26] L. Fang, X.M. Gao, X.L. Moore, H. Kiriazis, Y. Su, Z. Ming, Y.L. Lim, A.M. Dart and X.J. Du, Differences in inflammation, MMP activation and collagen damage account for gender difference in murine cardiac rupture following myocardial infarction, J Mol Cell Cardiol 43 (2007), 535-544.

[27] S.W. van den Borne, J.P. Cleutjens, R. Hanemaaijer, E.E. Creemers, J.F. Smits, M.J. Daemen and W.M. Blankesteijn, Increased matrix metalloproteinase- 8 and -9 activity in patients with infarct rupture after myocardial infarction, Cardiovasc Pathol 18 (2009), 37-43. 


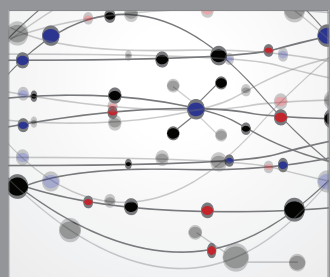

The Scientific World Journal
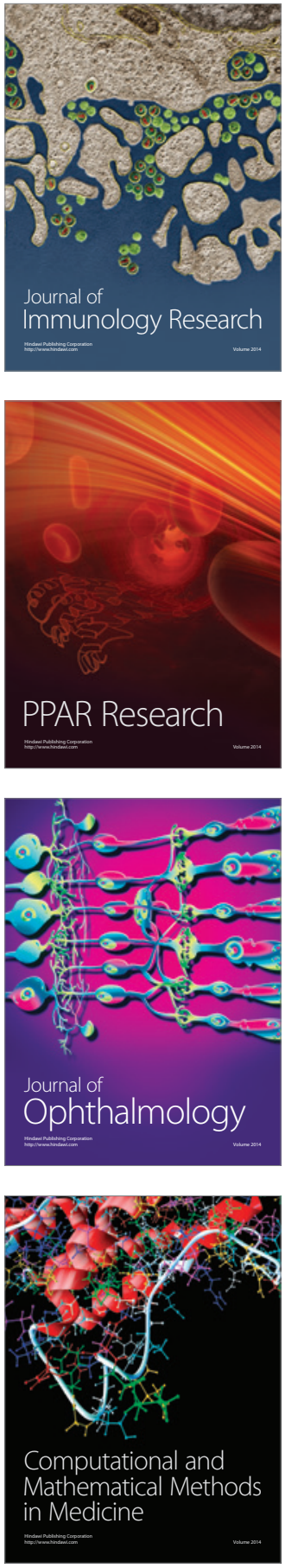

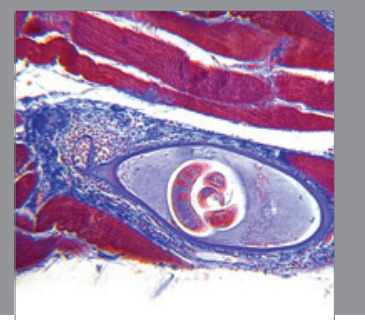

Gastroenterology

Research and Practice
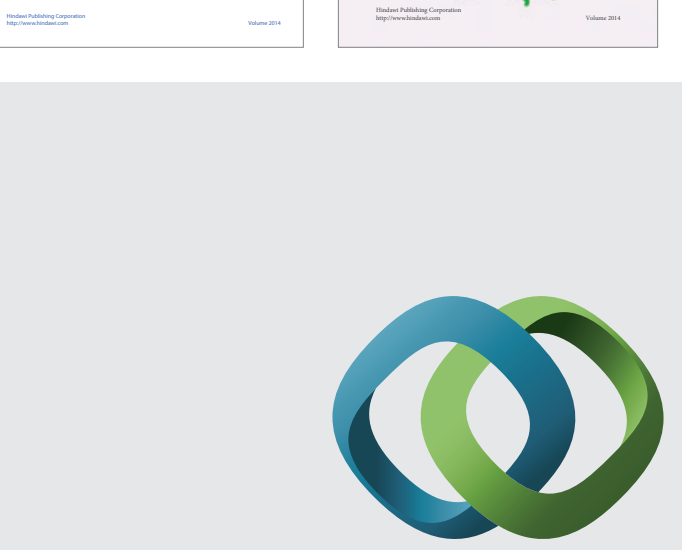

\section{Hindawi}

Submit your manuscripts at

http://www.hindawi.com
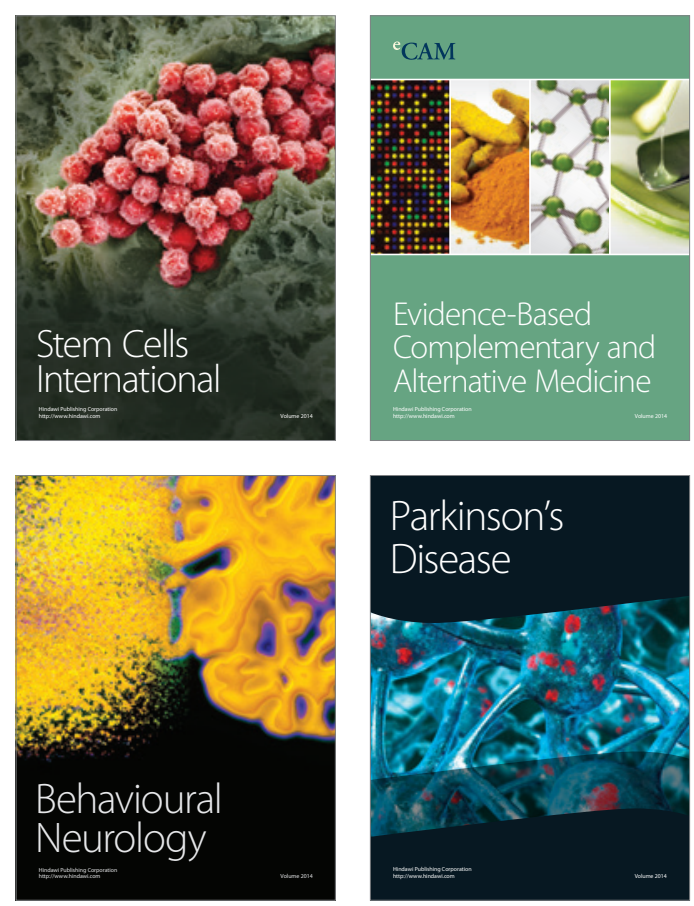

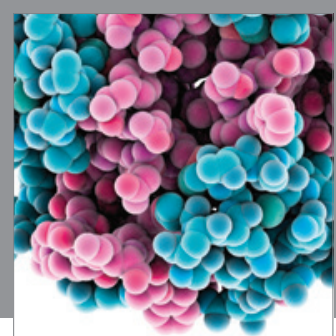

Journal of
Diabetes Research

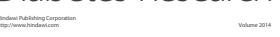

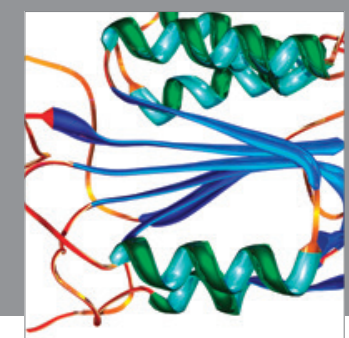

Disease Markers
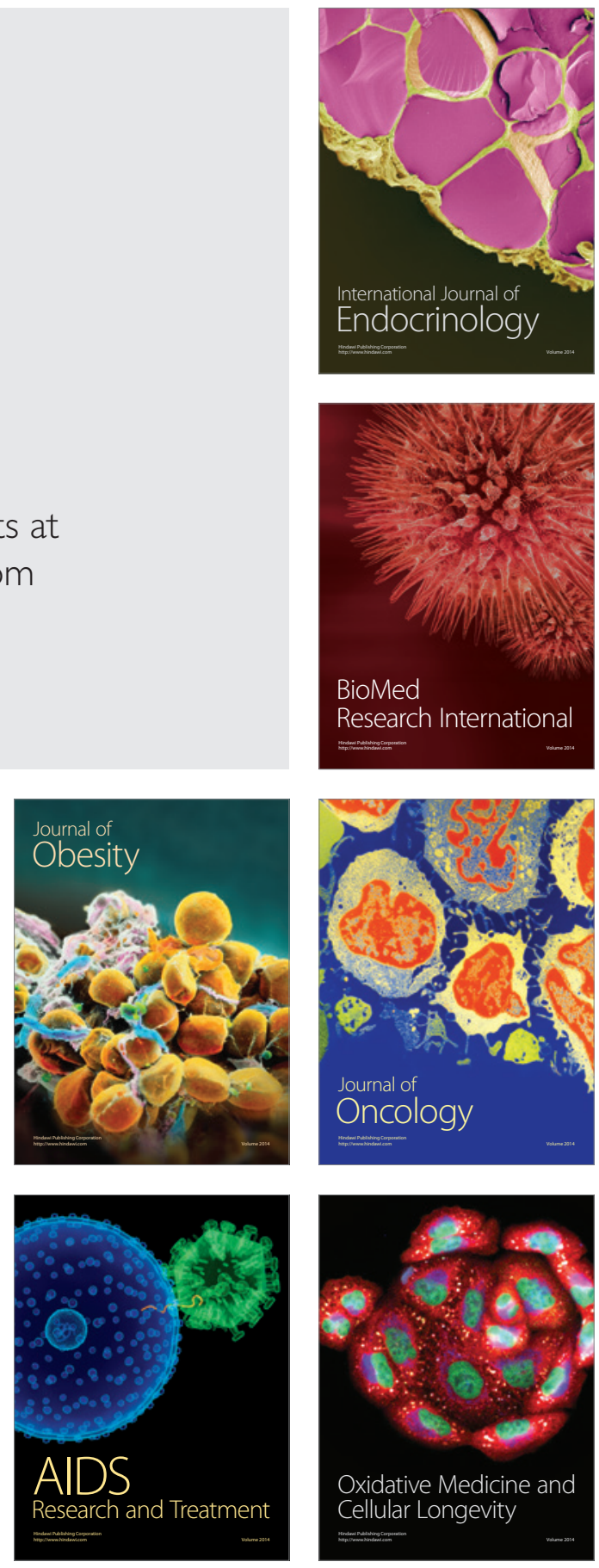